\title{
AVALIAÇÃO DE DOIS ESQUEMAS DE MONITORIZAÇÃO DOMICILIAR EM PACIENTES COM DIABETES MELLITUS DO TIPO $1^{1}$
}

\author{
EVALUATION OF TWO MONITORING SCHEMES IN TYPE 1 DIABETES \\ MELLITUS PATIENTS
}

\section{EVALUACIÓN DE DOS ESQUEMAS DE MONITORIZACIÓN DOMICILIAR EN PACIENTES COM DIABETES MELLITUS TIPO 1}

\author{
Sonia Aurora Alves Grossi * \\ Tamara Iwanow Cianciarullo** \\ Thais Della Manna***
}

Grossi SAA, Cianciarullo TI, Manna TD. Avaliação de dois esquemas de monitorização domiciliar em pacientes com diabetes mellitus tipo 1. Rev Esc Enferm USP 2002; 36(4): 317-23.

\begin{abstract}
RESUMO
O estudo teve por objetivo avaliar a efetividade de esquemas de monitorização domiciliar sangüineo e urinário, na obtenção de adequado controle glicêmico, em pacientes com diabetes mellitus do tipo 1, em regime quinzenal de ajuste terapêutico; durante 6 meses de participação em grupos educativos. A amostra foi de 34 pacientes divididos em dois grupos. Os pacientes do grupo A realizaram monitorização domiciliar da glicemia capilar 1 vez ao dia e os do grupo $\boldsymbol{B}$ realizaram monitorização domiciliar da glicosúria 1 vez ao dia, conforme esquemas preconizados. Estes esquemas possibilitaram construção de perfis e de ajustes terapêuticos. Os resultados mostraram que o uso sistemático dos testes domiciliares sangúineos e urinários da forma prescrita, não proporcionou melhora significante no controle metabólico em nenhum dos dois grupos. Entretanto, favoreceu o processo educativo e possibilitou reflexões sobre a necessidade de intensificação da monitorização glicêmica.
\end{abstract}

PALAVRAS-CHAVE: Diabetes mellitus tipo 1. Monitorização domiciliar de glicemia. Educação do paciente.

\section{ABSTRACT}

The goal of this study was to evaluate the effectiveness of two monitoring schemes(blood and urine) in the metabolic control of type $l$ diabetic patients, in biweekly therapeutic adjustments, along 6 months of participation in the educational groups. A sample of 34 patients was divided in two groups. The interventions proposed to group A were daily blood glucose monitoring, during three consecutive days for each period (before breakfast, before lunch, before dinner and before bed) and biweekly in the dawn. For the other group B was proposed daily urine glucose monitoring, during three consecutive days for each period (before breakfast, before lunch, before dinner and before bed). These schemes were used to construct glycemic profile and to determine the therapeutic adjustments. The results evidenced that there was no significant statistical difference in the metabolic control after proposed intervention in each group. In spite of this, the monitoring facilitated the educacional process and the considerations about the use of more intensive monitoring schemes.

KEYWORDS: Type 1 diabetes mellitus. Blood glucose self-monitoring. Patient education.

\section{RESUMIEN}

Este estudio tiene como objetivo evaluar la efectividad de dos esquemas de monitorización sanguineo y de orina a nivel domiciliar, para obtener un adecuado control glicémico en pacientes con diabetes mellitus tipo 1. El ajuste terapéutico se realizó a cada quince dias en el plazo de seis meses com la participación en grupos educativos. La muestra stubo formada por 34 pacientes divididos en dos grupos: los pacientes del grupo A realizaron monitorización domiciliar de la glicemia capilar una vez al dia y los del grupo B hicieron monitorización de la glicosuria también una vez al dia según esquemas pre-establecidos. Estos esquemas posibilitaron la construción de los perfiles y ajustes terapéuticos. Los resultados indicaron que el uso de los tests domiciliares sanguineos y orinarios pre-estabelecidos en los dos grupos, no proporcionaran una mejoría significativa en el control metabólico. Sin embargo, favoreció en el proceso educativo, posibilitando reflexiones sobre la necesidad de intensificar la monitorización glicémica en el domicilio.

PALABRAS -CLAVE: Diabetes mellitus tipo 1. Automonitorización de la glucose de la sangre. Educación del paciente.

\footnotetext{
1 O presente trabalho constitui parte da Tese de Doutorado "Avaliação de dois esquemas de monitorização domiciliar em pacientes com diabetes mellitus do tipo 1" apresentada à EEUSP no ano de 1999.

* Profa. Dra. do Departamento de Enfermagem Médico-Cirúrgica da Escola de Enfermagem da USP. (E-mail-sogrossi@usp.br)

**Profa. Titular do Departamento de Enfermagem Médico-Cirúrgica da Escola de Enfermagem da USP orientadora da Tese de Doutorado.

***Mestre em Medicina pela Faculdade de Medicina da Universidade de São Paulo, Médica Assistente da Unidade de Endocrinologia Pediátrica do. Instituto da Criança do Hospital das Clínicas da FMUSP.
} 


\section{INTRODUÇÃO}

O diabetes mellitus do tipo 1 , corresponde a $5 \%$ do total de casos de diabetes, é a forma mais comum entre crianças, adolescentes e adultos jovens e representa a segunda doença crônica mais comum nestas faixas etárias(1). Caracteriza-se pela perda da capacidade do pâncreas em produzir insulina por destruição completa das células beta das ilhotas de Langerhans ${ }^{(2-5)}$. A destruição auto imune das células beta pode ser evidenciada pela presença de anticorpos antiilhotas (ICA), anticorpos contra a enzima descarboxilase do ácido glutâmico (GAD) e anticorpos contra a própria insulina (IAA). Os fatores responsáveis por esta destruição são pouco conhecidos e embora as viroses e agentes químicos estejam sendo considerados como agentes desencadeantes iniciais da reação autoimune, agentes específicos são raramente identificados como causa do diabetes do tipo $1^{(3)}$. Nas pessoas acometidas deste tipo de diabetes, o tratamento insulínico é essencial à vida e previne a cetoacidose $(34)$

A partir da instalação da doença, desenvolvemse importantes distúrbios no metabolismo dos hidratos de carbono, lipídeos e proteínas que favorecem o aparecimento das complicações agudas e crônicas ${ }^{(6)}$. A importância do diabetes mellitus como um sério problema de saúde pública está no fato de que a maioria das complicações crônicas crônicas da doença é altamente incapacitante para a realização das atividades diárias e produtivas, compromete a qualidade de vida e o tratamento das mesmas é extremamente oneroso para o sistema de saúde $(7,8)$. Ao longo das últimas décadas, inúmeras pesquisas objetivando melhor controle metabólico, têm sido realizadas e novas tecnologias incorporadas ao tratamento do diabetes. A monitorização da glicosúria, amplamente utilizada nas décadas passadas e menos utilizada nos dias de hoje, principalmente entre os diabéticos do tipo 1 , foi a primeira tentativa de avaliação do controle glicêmico domiciliar, entretanto apresenta limitações importantes no controle glicêmico. A superioridade da monitorização da glicemia em relação à glicosúria na viabilidade de obtenção de melhor controle metabólico é documentada em muitos estudos(9_16). Sabe-se entretanto que, se a monitorização da glicemia não fizer parte de um plano que implique em maior envolvimento dos pacientes com a equipe ou quando os resultados da automonitorização são subtilizados para a adequação terapêutica, não ocorre melhora no controle glicêmico, indicando desta forma que a monitorização por si só não é efetiva(10,13,17)

Evidências a partir de observações clínicas, epidemiológicas e bioquímicas indicam que a manutenção de parâmetros glicêmicos e da pressão arterial próximos à normalidade podem reduzir a incidência e a severidade das complicações neuropáticas, macro e microvasculares ${ }^{(18-20)}$. Nos últimos anos, muitos questionamentos, reflexões e estudos têm sido feitos a respeito das dificuldades na implementação dos rígidos controles preconizados pelo DCCT e também sobre suas implicações na prática clinica, nos programas educacionais, no autocontrole, na qualidade de vida e no custo do tratamento, especialmente nos serviços que não dispõem de recursos e de profissionais capacitados.

Assim sendo, pretendeu-se por meio deste estudo, avaliar a efetividade de dois esquemas de monitorização domiciliar sangüíneo e urinário, na obtenção de adequado controle glicêmico, em pacientes com diabetes mellitus do tipo 1 , em regime quinzenal de ajuste terapêtico; ao longo de 6 meses de participação em grupos educativos.

\section{CASUÍSTICA E MÉTODO}

Esta pesquisa caracteriza-se pela utilização de um projeto quase-experimental pois envolve a manipulação de variáveis independentes, isto é, a instituição de intervenções e uma amostragem de conveniência(21).

O estudo foi realizado no Ambulatório da Liga de Controle do Diabetes e no Ambulatório do Instituto da Criança, ambos pertencentes ao Hospital das Clínicas da Faculdade de Medicina da Universidade de São Paulo(HCFMUSP). A autorização prévia para a realização do trabalho consta do parecer sobre o protocolo de pesquisa $n^{\circ} .144 / 98$ da Comissão de Ética para Análise de Projetos de Pesquisa da Diretoria Clínica do HCFMUSP.

A população foi constituída de pacientes com diabetes mellitus do tipo 1 que atenderam os seguintes critérios de inclusão: idade não inferior a 2 anos(pela maior vulnerabilidade às infecções respiratórias, as quais elevam os niveis glicêmicos plasmáticos), condições de realizar monitorização sanguínea ou urinária 1 vez ao dia pelo periodo de 6 meses e concordância em participar de 13 reuniões educativas quinzenais neste mesmo período. Os critérios de exclusão da população foram: pacientes com menos de um ano de tempo de doença, aqueles em uso esporádico ou contínuo de medicamentos hiperglicemiantes e pacientes ou cuidadores que não concordaram em participar do estudo.

A amostra foi inicialmente constituída de 34 pacientes, os quais foram divididos em dois grupos de 17 pacientes, a saber: grupo A e grupo B. A quantificação da amostra foi determinada após teste piloto por meio do teste de aderência de Kolmogorov-Smirnov para 22 resultados de hemoglobina glicosilada. Os grupos A e B foram pareados de acordo com os resultados da hemoglobina glicosilada (HbAlc). 
Os pacientes do grupo A realizaram monitorização e registro domiciliar da glicemia capilar 1 vez ao dia, durante 3 dias consecutivos em cada um dos horários preconizados (antes do café, antes do almoço, antes do jantar e antes de deitar) e quinzenalmente às 3 horas da madrugada, durante o período de 6 meses. Os pacientes do grupo B realizaram monitorização e registro domiciliar da glicosúria $1 \mathrm{vez}$ ao dia, durante 3 dias consecutivos em cada um dos horários preconizados (antes do café, antes do almoço, antes do jantar e antes de deitar) durante 6 meses.Os pacientes do Grupo A e B participaram de 13 reuniões educativas distintas que foram realizadas quinzenalmente. As reuniões educativas tiveram como objetivos fornecer informações sobre aspectos importantes no manejo do diabetes, esclarecer as dúvidas emergentes do grupo, analisar conjuntamente o perfil glicêmico no grupo A ou o perfil das glicosúrias no grupo B, de um dos participantes do grupo e, proceder os ensaios sobre os ajustes terapêuticos. Ao final das reuniões, a equipe procedia aos ajustes terapêuticos individuais e efetuava as orientações necessárias (insulina, dieta e atividade fisica).

As dosagens de HbAlc neste estudo foram realizadas pelo método de captura iônica IMx Abbott, tendo como faixa de normalidade os valores entre 5,5 a $8,5 \%$.

Com relação à monitorização da glicemia capilar, os pacientes foram formalmente reorientados sobre a técnica correta de monitorização domiciliar da glicemia capilar. Os testes foram realizados com os monitores de glicemia e tiras reagentes do "Sistema Advantage"da Boheringer Mannheim Corporation. A determinação da qualidade dos controles das glicemias foi feita tendo-se como referência as recomendações da American Diabetes Association(22-25). Desta forma, os resultados glicêmicos de antes do café da manhã e da madrugada foram considerados bons quando estiveram na faixa compreendida entre $80-120 \mathrm{mg} /$ dl, satisfatórios quando na faixa entre $121-140 \mathrm{mg} /$ dl e ruins quando menores do que $80 \mathrm{mg} / \mathrm{dl}$ e maiores que $140 \mathrm{mg} / \mathrm{dl}$. Os resultados glicêmicos de antes do almoço e antes do jantar foram considerados bons quando estiveram na faixa entre $80-160 \mathrm{mg} / \mathrm{dl}$, satisfatórios quando na faixa entre $161-180 \mathrm{mg} / \mathrm{dl} \mathrm{e}$ ruins quando menores do que $80 \mathrm{mg} / \mathrm{dl}$ e maiores do que $180 \mathrm{mg} / \mathrm{dl}$. Os resultados glicêmicos de antes de deitar foram considerados bons quando compreendidos na faixa entre 101 a $140 \mathrm{mg} / \mathrm{dl}$, satisfatórios quando na faixa entre 141 - 160 e ruins quando menores do que $100 \mathrm{mg} / \mathrm{dl}$ e maiores do que $180 \mathrm{mg} / \mathrm{dl}$.

Em relação a monitorização da glicosúria os pacientes foram formalmente orientados sobre a técnica correta de monitorização domiciliar da glicosúria, no primeiro dia da reunião educativa. Os testes foram realizados com as tiras reagentes "Diastix" da Bayer Diagnóstica, que permitem uma análise semi-quantitativa da glicose urinária. A determinação da qualidade dos controles das glicosúrias foi feita considerando-se a ausência ou presença de traços de glicose na urina como o padrão de normalidade.

Como parâmetro de controle foi utilizado o controle histórico, de forma que, cada paciente foi controle de si mesmo no período de 6 a 12 meses anteriores ao início do estudo, com relação aos resultados de hemoglobina glicosilada(HbAlc). Assim sendo, tivemos 6 resultados de HbAlc, de cada paciente sendo, 3 antes das intervenções propostas e 3 após as mesmas.

O estudo foi realizado com crianças e adolescentes, juntamente com seus cuidadores (pais ou responsáveis), os quais foram esclarecidos quanto aos objetivos, procedimentos, riscos e benefícios da investigação. O termo de consentimento pósinformação (Resolução $n^{\circ} .1$ de 13/06/1988-CNS), padronizado pela Comissão de Ética do HCFMUSP, foi assinado por todos os pacientes ou seus representantes legais.

No decorrer do estudo, houve nove desistências no grupo B. Assim sendo, a amostra ficou constituída de 25 pacientes, sendo 17 pacientes no grupo A e 8 pacientes no grupo B. Em ambos os grupos a faixa etária predominante foi a de adolescentes sendo 7 $(41,17 \%)$ no grupo A e $5(62,50 \%)$ no grupo B, seguido dos escolares sendo $6(35,29 \%)$ no grupo A e $3(37,50 \%)$ no grupo B. Em relação ao sexo, observou-se que, o sexo feminino $(64,73 \%)$ foi predominante no grupo A, enquanto o masculino foi $(75 \%)$ predominante no grupo B. Com relação ao tempo de diabetes, $82,34 \%$ dos constituintes do grupo A e 100\% constituintes do grupo B possuíam diabetes há menos de 9 anos. No grupo A, $11,76 \%$ tinham a doença entre 9 e 12 anos e $5,89 \%$ há mais de 12 anos. O tratamento insulinoterápico da amostra estudada caracterizouse pelo uso de insulina de ação intermediária (NPH) usada isoladamente ou associada com insulina de ação rápida $(R)$. As doses variaram de 1 a 2 ao dia, feitas antes do café da manhã (Ac) e antes do jantar (Aj). O uso de insulina de ação ultra rápida (lispro) era feito por apenas 1 paciente, antes do jantar, quando a glicemia estivesse maior do que $180 \mathrm{mg} / \mathrm{dl}$. Este mesmo paciente fazia uso de dose única de NPH-Ac. A maioria dos pacientes do grupo A $(70,59 \%)$ e do grupo B $(62,5 \%)$ recebiam insulinoterapia de 2 doses diárias e 41,19\% dos pacientes do grupo A e 50\% do grupo B recebiam associação de insulina de ação intermediária e regular. Portanto $58,81 \%$ dos pacientes no grupo A e $50 \%$ dos pacientes do grupo B, somente utilizavam insulina NPH para o controle metabólico. Com relação à quantidade de insulina administrada por $\mathrm{Kg}$ de peso, no grupo A, 58,82\% dos pacientes tomavam menos de $1 \mathrm{U} / \mathrm{Kg} /$ dia de insulina e que $41,18 \%$ tomavam entre 1,01 e $1,5 \mathrm{U} / \mathrm{Kg} /$ dia. A maioria dos pacientes do grupo 
B, $(87,50 \%)$ tomavam menos de $1 \mathrm{U} / \mathrm{Kg} /$ dia. Quanto ao peso corporal a maioria da amostra encontrava-se eutrófica. No grupo A, 2 pacientes $(11,76 \%)$ estavam com sobrepeso e $1(5,89 \%)$ com baixo peso. A maioria dos pacientes do grupo A $(70,59 \%)$ encontrava-se na faixa de HbAlc compreendida entre 9 e $11 \%$. No grupo B, $50 \%$ dos pacientes apresentavam-se com a HbAlc entre 11 e $13 \%, 25 \%$ entre 13 e $15 \%$ e $25 \%$ entre 7 e 9 $\%$. Considerando que a faixa de normalidade para o método IMx-Abbot varia entre 5,5 e $8,5 \%$, pode-se afirmar que, a maioria dos pacientes apresentavam os resultados elevados e que, os constituintes do grupo A, estavam em melhor controle metabólico pois, 15 ( $88,23 \%$ ) tinham $\mathrm{HbA} 1 \mathrm{c}$ entre 7 e $11 \%$ enquanto que apenas $2(25 \%)$ do grupo B estavam nesta faixa. Por outro lado a maioria dos pacientes do grupo B $(75 \%)$ tinham HbAlc entre 11 e $15 \%$. A heterogeneidade entre os dois grupos deveu-se ao fato que, embora os grupos tivessem sido pareados pelos resultados das HbA1c no início do estudo, houve 9 desistências no grupo B. A comparação da média das 3 análises de HbAlc anteriores ao estudo, por meio do teste $t$, caracteriza a heterogeneidade entre os grupos com relação ao controle metabólico, ao início do estudo $(\mathrm{p}<0,001)$.

Todas as provas estatísticas foram realizadas admitindo-se um erro de primeira espécie de $5 \%$. 0 tratamento estatístico teve como objetivo a comparação de dados intergrupos e intragrupos (A e B) e com os parâmetros de controle. As provas não paramétricas utilizadas foram: teste de aderência de Kolmogorov-Smirnov que foi empregado para a determinação inicial do tamanho da amostra; prova de associação do Qui-quadrado que foi empregada para testar a associação entre o número de testes glicêmicos e de glicosúrias (previstos e realizados), e grupos (A e B), durante o tempo do estudo. As provas paramétricas utilizadas foram:teste $t$ o qual foi empregado para identificar as possiveis diferenças significantes entre as médias das $\mathrm{HbA} 1 \mathrm{c}$ dos grupos $\mathrm{A}$ e $\mathrm{B}$ antes do início do estudo e também para identificar as diferenças significantes com relação à presença às aulas nos grupos A e B; análise de variância para medidas repetidas: foi empregada para a comparação das médias das HbA1c, antes e após as intervenções, no grupo A.

\section{RESULTADOS E DISCUSSÃO}

Os esquemas regulares de monitorização domiciliares da glicemia e glicosúria, propostos neste estudo, foram incorporados à vida diária dos pacientes, sem grandes dificuldades, ao longo dos 6 meses de estudo. No grupo A, dos 3587 testes sangüineos propostos, $3259(90,85 \%)$ foram realizados. No grupo B, mesmo com menor adesão, dos 1560 testes urinários propostos, $1173(75,19 \%)$ foram feitos. A prova de associação do Qui quadrado evidenciou que a adesão no grupo A foi maior em relação ao grupo $B(p<0,001)$. A maior adesão à monitorização sangüínea pode estar relacionada a maior motivação para o autocontrole apresentada pelo grupo A, deste o início deste trabalho. Todos os participantes permaneceram até o final do estudo, a presença nos 13 reuniões educativas foi de 11,94 vezes e as poucas faltas sempre foram justificadas. No grupo B a presença às reuniões foi de 9,56 vezes sendo a diferença estatisticamente significante entre os dois grupos $(p=0,0228)$. As faltas não foram justificadas e houve 9 desistências, como já foi citado anteriormente. A ocorrência significativamente maior de hipoglicemias entre os pacientes do grupo A, também pode ter sido um dos fatores de maior adesão. A vivência desagradável e perigosa ocasionada pelos baixos níveis de glicose sangüínea pode ter contribuído para a adoção deste comportamento direcionado ao autocuidado. A monitorização da glicemia com regularidade, permite a análise da freqüência, severidade das hipoglicemias e da labilidade dos níveis glicêmicos rotineiros, fatores estes preditivos de hipoglicemias mais severas(26). Além disso, estudos realizados com crianças e adolescentes, mostram que a monitorização sangüinea é preferida em relação a monitorização urinária pois é mais confiável, facilita a tomada de decisões e favorece o senso de controle(14,27_29). Na nossa experiência é comum observar que os jovens sentem-se muitos estimulados pelo fato de utilizarem um equipamento eletrônico que fornece resultados imediatos sobre seu controle metabólico. Salienta-se ainda que, o grupo A é predominantemente feminino, e como salientam Thompson( ${ }^{30}$, as mulheres e os jovens demonstram mais desejo de controlar suas glicemias.

Os esquemas de monitorização propostos neste estudo viabilizaram a construção de perfis quinzenais individuais. Os encontros educativos possibilitaram a discussão grupal da interação entre estes perfis com os picos de ação da insulina, dieta e atividade física. Desta forma a monitorização favoreceu o processo educativo voltado para o enfrentamento e resolução dos problemas práticos e reais da vida diária de cada um. O conhecimento advindo deste tipo de experiência confere segurança e autonomia para a tomada de decisões e quanto maior a participação do paciente e familiar neste processo maior é adesão ao autocuidado (12,13,31,32). Embora a proposta de monitorização neste estudo tenha tido como objetivo fundamental usar sistematicamente os resultados dos testes domiciliares no plano terapêutico dos ajustes quinzenais, visando a melhoria do controle metabólico, os resultados encontrados não evidenciaram esta melhoria, como pode ser visto a seguir: 
Tabela 1- Média e desvio padrão das HbA1c antes e após as intervenções, no grupo A. São Paulo, 1999.

\begin{tabular}{ccccccc}
\hline Momentos & $\mathbf{1}$ & $\mathbf{2}$ & $\mathbf{4}$ & $\mathbf{4 b A 1 c}$ & $\mathbf{5}$ & $\mathbf{6}$ \\
$\begin{array}{c}\mathrm{n}^{\circ} \mathrm{de} \\
\text { pacientes }\end{array}$ & $\mathrm{n}=15$ & $\mathrm{n}=16$ & $\mathrm{n}=17$ & $\mathrm{n}=16$ & $\mathrm{n}=17$ & $\mathrm{n}=17$ \\
\hline $\begin{array}{c}\text { Média } \\
\begin{array}{c}\text { Desvio } \\
\text { padrão }\end{array}\end{array}$ & $\mathbf{1 1 , 9 9}$ & $\mathbf{1 0 , 6}$ & $\mathbf{9 , 4 4}$ & $\mathbf{9 , 9 6}$ & $\mathbf{1 0 , 4}$ & $\mathbf{1 0 , 0}$ \\
\hline
\end{tabular}

A tabela 1 mostra a média das HbA1c dos 17 pacientes pertencentes ao grupo A, realizadas antes das intervenções propostas neste estudo $(1,2,3)$ e após as intervenções $(4,5,6)$. 0 desvio padrão reflete a homogeneidade dos valores encontrados dentro do grupo em todos os 6 momentos.

Dos 17 pacientes pertencentes a este grupo, 2 deles não tiveram a totalidade das dosagens de HbA1c previstas para o estudo. Desta forma, uma análise comparativa das médias das HbA1c antes e após as intervenções só foi possivel ser realizada com 15 pacientes.

Gráfico 1 - Média das HbA1c antes e após as intervenções no grupo A. São Paulo, 1999.

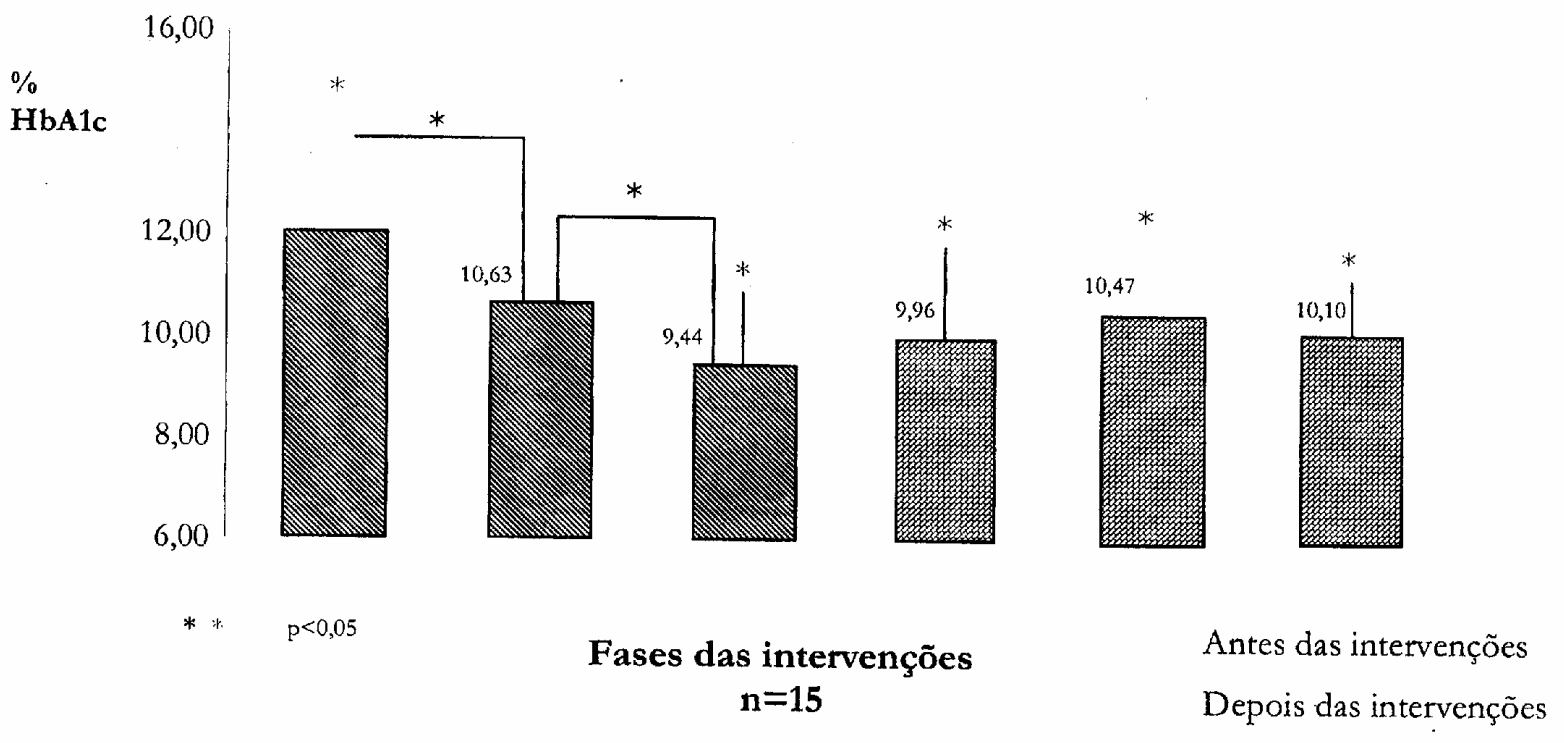

O gráfico 1 , mostra a média das $\mathrm{HbA} 1 \mathrm{c}$ dos 15 pacientes, nos momentos 1,2 e 3 , antes do início do estudo e nos momentos 4, 5 e 6 , ao longo dos 6 meses do estudo, no grupo A $(\mathrm{n}=15)$. A análise de variância para medidas repetidas evidenciou que quando a média da HbA1c no momento $1(11,99 \%)$ foi comparada às médias dos momentos $2(10,63 \%), 3(9,44 \%), 4$ $(9,96 \%), 5(10,47 \%)$ e $6(10,10 \%)$ houve diferença estatisticamente significativa $(p<0,05)$ em todas as comparações realizadas. O mesmo teste foi aplicado para os momentos 2 e 3 ( $p=0,0009), 3$ e $4(p=0,27)$, 4 e $5(p=0,69)$ e 5 e $6(p=0,07)$. A queda nos valores de HbA1c entre os momentos 2 e 3 , também foi estatisticamente significativa $(p<0,001)$. Entretanto, a comparação entre os momentos 3 e 4,4 e 5,5 e 6 não se apresentou com diferença estatisticamente significativa. Apesar disso, embora a média das HbA1c tenha se mantido em niveis superiores ao desejado, os ajustes terapêuticos viabilizados pelos perfis glicêmicos quinzenais mantiveram os niveis glicêmicos estáveis durante o período do estudo. Uma melhora significativa do controle metabólico pode ser observada nos parâmetros de controle, fato este que pode ser explicado pela reestruturação nos processos de trabalho desenvolvidos pela equipe do serviço do qual fazemos parte. A enfermagem foi introduzida neste serviço, que até então contava somente com a equipe médica para o atendimento dos pacientes e familiares, por meio de consulta individualizada. Desde então, ações educativas individualizadas e informais realizadas nas consultas de enfermagem, o incentivo à aquisição de monitores de glicemia e à realização da monitorização domiciliar passaram a ser o foco das atividades de enfermagem. Foi então realizado um estudo com o objetivo de avaliar a influência dessas ações educativas e do incentivo à monitorização domiciliar da glicose sangüínea no controle metabólico. Os resultados mostraram que a média das HbA1c anteriores ao início das atividades de enfermagem $(12,76 \%)$ foi significativamente maior quando 
comparada com a média das HbA1c $(11,08 \%)$ após o desenvolvimento dessas atividades $(\mathrm{p}<0,01)(33,34)$. Embora caracterizada a heterogeneidade, com relação ao controle metabólico, entre os grupos A e B ao início do estudo, heterogeneidade esta mantida ao longo do estudo $(\mathrm{p}<0,001)$, não se pode deixar de considerar que os pacientes que realizaram a monitorização sangüinea tiveram ajustes insulinoterápicos mais agressivos, e se mantiveram em melhor controle glicêmico durante todo o tempo, além de estarem mais motivados para a alcance de adequado controle metabólico.

Resultados semelhantes aos encontrados neste estudo, foram descritos por Mann et al(35), onde os autores não encontraram variações significativas no controle metabólico entre grupos de pacientes que realizaram monitorização sangüínea ou urinária. Entretanto eles relatam que a monitorização glicêmica reduziu o número de hospitalizações por cetoacidose diabética.

Starostina et al(36), num estudo prospectivo com 120 pacientes diabéticos do tipo 1 em seguimento por 2 anos, avaliaram a efetividade de esquemas de monitorização glicêmica e urinária sobre o controle metabólico e concluíram que nos dois grupos houve queda significativa da HbAlc.

A capacidade da monitorização sangüinea em proporcionar melhores índices glicêmicos foi demonstrada em muitos estudos $(10,12,14)$ Entretanto alguns trabalhos salientam que o bom controle metabólico é independente do tipo ou freqüência da monitorização domiciliar e que, ao longo do tempo, as melhoras obtidas nos níveis glicêmicos com qualquer tipo de monitorização, tendem a se deteriorar se não houver acompanhamento, atenção intensiva $e$ envolvimento da equipe com os o pacientes $(27,36,37,38$ Estudos mais recentes salientam a necessidade de intensificação da monitorização sangüínea, de forma que o perfil glicêmico seja mais sensivel as flutuações glicêmicas diárias e permita maior exatidão dos ajustes terapêuticos $(14,17,20,29)$ Quando esta intensificação atinge o número de 4 vezes ao dia e os resultados são utilizados para o ajuste diário nas múltiplas doses de insulina o controle metabólico é seguramente otimizado(18,20). A tentativa de melhorar o controle metabólico dos pacientes no nosso serviço por meio do incentivo à monitorização sangüínea 1 vez ao dia foi bem sucedida no momento anterior a este estudo. Entretanto, a idéia de que a implementação sistemática deste esquema, como parte do processo educativo e do planejamento terapêutico pudesse melhorar ainda mais os niveis glicêmicos, não foi confirmada por meio deste trabalho. E necessário salientar ainda que, a abordagem dietética realizada nos programas educativos foi sempre qualitativa, e isto pode ter contribuído com os resultados.
Tabela 2 - Média e desvio padrão das HbA1c antes e após as intervenções, no grupo B. São Paulo, 1999.

\begin{tabular}{|c|c|c|c|c|c|c|}
\hline \multirow[b]{2}{*}{ Momentos } & \multicolumn{6}{|c|}{ HbA1c } \\
\hline & 1 & 2 & 3 & 4 & 5 & 6 \\
\hline $\begin{array}{c}\mathrm{n}^{\circ} \mathrm{de} \\
\text { pacientes }\end{array}$ & $\mathrm{n}=8$ & $\mathrm{n}=6$ & $n=6$ & $\mathrm{n}=5$ & $\mathrm{n}=8$ & $\mathrm{n}=8$ \\
\hline $\begin{array}{l}\text { Média } \\
\text { Desvio } \\
\text { padrão }\end{array}$ & $\begin{array}{l}13,4 \\
2,85\end{array}$ & $\begin{array}{l}13,2 \\
3,05\end{array}$ & $\begin{array}{l}11,9 \\
2,56\end{array}$ & $\begin{array}{l}12,8 \\
2,85\end{array}$ & $\begin{array}{l}12,6 \\
4,62\end{array}$ & $\begin{array}{l}11,5 \\
1,94\end{array}$ \\
\hline
\end{tabular}

A tabela 2 mostra a média das $\mathrm{HbA} 1 \mathrm{c}$ dos pacientes pertencentes ao grupo B, nos 3 momentos anteriores às intervenções propostas neste estudo $(1,2,3)$ e, nos 3 momentos após as intervenções $(4,5,6)$. 0 desvio padrão evidencia a homogeneidade dos valores encontrados dentro do grupo em todos os 6 momentos analisados. Observa-se também que a média das HbA1c do momento 6, ao final do estudo, apresentou similaridade com a média das HbAlc do momento 3, do início do estudo. Dos 8 pacientes pertencentes a este grupo, apenas 3 deles obtiveram a totalidade das dosagens de HbA1c previstas no estudo. Assim sendo, não foi viável a realização de uma análise estatística comparativa antes e após as intervenções, neste grupo de pacientes.

\section{CONCLUSÃO}

Neste estudo, o uso sistemático dos resultados dos testes domiciliares sangüíneos e urinários prescritos, como parte do processo educativo e do planejamento terapêutico quinzenal, não proporcionou melhora significativa no controle metabólico em nenhum dos dois grupos do estudo. No grupo A, no qual foi possivel aplicar o teste de análise de variância para medidas repetidas, não houve diferença estatisticamente significativa na média das $\mathrm{HbA1c}$ antes e após as intervenções implementadas $(p>0,05)$. No grupo B, não foi possivel a realização de análise estatística comparativa entretanto, os resultados das médias das HbA1c antes e após as intervenções apresentaram similaridade.

\section{REFERÊNCIAS BIBLIOGRÁFICAS}

(1) Sociedade Brasileira de Diabetes. Consenso brasileiro de conceitos e condutas para o diabetes mellitus recomendações da Sociedade Brasilieira de Diabetes para a prática clinica. São Paulo; 1997.

(2) Ginsberg BH, Parkes JL. Etiologia do diabetes mellitus insulino-dependente. Terapêutica Diabetes1993; 1(1): 1-4.

(3) Bennett, PH. Definition, diagnosis and classification of diabetes mellitus and impaired glucose tolerance. In: Kahn CR, Weir GC, editors. Joslin's diabetes mellitus. $13^{\mathrm{a}}$.ed. Philadelphia: Lea \& Febiger; 1994. p. 193-200. 
(4) Malone E. Lessons for pedriaticians from the Diabetes Control and Complications Trial. Pediatr. Ann1994; 23(6): 295-9.

(5) Lerário AC. Nova classificação e critérios para o diagnóstico do diabetes mellitus. Diabetes Metab1997; 1(2): 65-7.

(6) Beland I, Passos JY. Clinical nursing: pathophysiological and psychosocial approaches. New York: Macmillan; 1981. Problems associated with diabetes mellitus; p.1037-77.

(7) Vinicor F. Is diabetes a public-health disorder? Diabetes Care 1994; 17: 22-7, Supplement. 1.

(8) Dagogo-Jack S. DCCT results and diabetes care in developing countries. Diabetes Care 1995; 18(3):416-7.

(9) Schiffrin A, Belmonte M. Multiple daily self-monitoring: its essential role in long-term glucose control in insulindependent diabetc patients treated with pump and multiple subcutaneous injections. Diabetes Care 1982; 5(5): 479-84.

(10) Carney RM, Schechter K, Homa M, Levandoski L, White $\mathrm{N}$, Santiago J. The effects of blood glucose testing versus urine sugar testing on the metabolic control of insulindependent diabetic children. Diabetes Care 1983; 6(4): 37880.

(11) Geffner ME, Kaplan SA, Lippe BM, Scott ME. Self-monitoring of blood glucose levels and intensified insulin therapy: acceptability and efficacy in childhood diabetes. JAMA1983; 249(21): 2913-6.

(12) Loman D, Galgani C. Monitoring diabetic children's blood glucose levels at home. MCN Am J Matern Child Nurs1984; 9(3): 192-6.

(13) Daneman D, Siminerio L, Transue D, Betschart J, Drash A, Becker D. The role of self-monitoring of blood glucose in the routine management of children with insulin-dependent diabetes mellitus. Diabetes Care 1985; 8(1):1-4.

(14) Wang P, Lau J, Chalmers TC. Meta-analysis of effects of intensive blood glucose control on late complications of type I diabetes. Lancet1993; 341(8856): 1306-9.

(15) Wolfsdorf JI, Anderson BJ, Pasquarello C. Treatment of the child with diabetes. In: Kahn, C. R.; Weir, G. C, editors. Joslin's diabetes mellitus. 13.ed. Philadelphia, Lea \& Febiger, 1994. p. 530-51.

(16) Cox DJ, Gonter-Frederick L, Polonski W, Schlundt D, Julian D, Clarke W. A multicenter evaluation of blood glucose awareness training-II. Diabetes Care 1995; 8(4): 523-8.

(17) Delamater AM, Bubb J, Dauis SG, Smith JA, Schmidt L, White NH, Santiago JV. Randomized prospective study of self-management training with newly diagnosed diabetic children. Diabetes Care 1990; 13(5): 492-8.

(18) Diabetes Control and Complications Trial Research Group. The effect of intensive treatment of diabetes on the development and progression of long-term complications in insulin-dependent diabetes mellitus. N Engl J Med1993; 329(14): 977-86.

(19) Eastman RC, Gorden P. The DCCT implications for diabetes treatment. Diabetes Reviews 1995; 2(3): 263-9.

(20) Charbonnel B, Boivineau C, Chopinet P, Daninos JM, Drounin D, Guyon F, Passa P Auto-surveillance glycémique chez le diabétique. Recommandations de L'Alfediam. Diabete Metab1995; 21(4), 285-9.

(21) Polit DF, Hungler BP. Fundamentos de pesquisa em enfermagem. 3. ed. Porto Alegre: Artes Médicas; 1995. Delineamento de pesquisa; 107-40.

(22) American Diabetes Association. Self monitoring of blood glucose. Diabetes Care1996a; 19 (Suppl 1): 62-6.
(23) American Diabetes Association. Standards of medical care for patients with diabetes mellitus. Diabetes Carel996b; 19 (Suppl 1): 8-15.

(24) American Diabetes Association. Standards of medical care for patients with diabetes mellitus. Diabetes Care1997; 20 (Supp1 1): 5-13.

(25) American Diabetes Association. Standards of medical care for patients with diabetes mellitus. Diabetes Care 2000; 23 (Suppl 1): 32-42.

(26) Cox DJ, Kovatchev BP Julian DM, Gonder-Frederick LA, Polonski WH, Schlundt DG, Clarke WL. Frequency of severe hypoglycemia in insulin-dependent diabetes mellitus can be predicted from self-monitoring blood glucose data. J Clin Endocrinol Metab1994; 79(6): 1659-62.

(27) Miller PFW, Stratton C, Tripp JH. Blood testing compared with urine testing in the long term control of diabetes. Arch Dis Child1983; 58(4): 294-7.

(28) Hermansson G, Ludvigsson J, Larsson Y. Home blood glucose monitoring in diabetic children and adolescents: a 3 year feasibility study. Acta Paediatr Scand1986; 75(1): 98-105.

(29) Pieber TR, Brunner GA, Schnedl WJ, Schattenberg S, Kaufmann P, Krejs GJ. Evaluation of structured outpatient group education program for intensive insulin therapy. Diabetes Care 1995; 18(5): 625-30.

(30) Thompson CJ, Cummings JF, Chalmers J, Gould C, Newton RW. How have patients reacted to the implications of the DCCT ? Diabetes Care 1996; 19(8): 876-79.

(31) Maldonato A, Bloise D, Ceci M, Fraticelli E, Fallucca E Diabetes mellitus: lessons from patient education. Patient Educ Couns 1995; 26(1/3): 57-66.

(32) Golin CE, Dimatteo MR, Gelberg L. The role of patient participation in the doctor visit. Diabetes Carel996; 19(10): 1153 -64.

(33) Mann NE Noronha JL, Johnston DL A prospective study to evaluate the benefits of long-term self-monitoring of blood glucose in diabetic children. Diabetes Care 1984; 7(4): 322-6.

(34) Grossi SAA. Monitorização domiciliar da glicemia em pacientes com diabetes mellitus insulino-dependente: relato de experiência. Pediatria1998a; 20(2): 154-60.

(35) Grossi SAA. Uma proposta de avaliação para pacientes com diabetes mellitus insulino-dependente.In: Programa do $3^{\circ}$ Congresso Paulista de Diabetes e Metabolismo; 1998; Aguas de Lindóia.São Paulo; 1998b. p.28.

(36) Starostina EG, Muhlhauser I, Antsiferov M, Galstyan GR, Trautner C, Jorgens V, Bott U, Berger M, Dedov II. Effectiveness and cost-benefit analysis of intensive treatment and teaching programmes for type 1 (insulin-dependent) diabetes mellitus in Moscow - blood glucose versus urine glucose selfmonitoring. Diabetologia1994; 37(2): 170-6.

(37) Worth R, Home PD, Johnston DG, Anderson J, Ashworth L, Burrin JM et al. Intensive attention improves glycaemic control in insulin-dependent diabetes without further advantage from home blood glucose monitoring: results of a controlled trial. Br Med J(Clin Res Ed) 1982; 285(6350): 1233-40.

(38) Wing RR, Lamparski DM, Zaslow S, Betschert J, Siminerio L, Becker D. Frequency and accuracy of self-monitoring of blood glucose in children: reltionship to glicemic control. Diabetes Carel985; 8(3): 214-18.

Artigo recebido em 04/03/02 Artigo aprovado em 20/09/02 